\title{
Phidias numbers as a basis for Fibonacci analogues
}

\author{
P. S. Kosobutskyy \\ Department of Computer-Aided Design \\ Lviv Polytechnic National University \\ S. Bandery 12 St., Lviv, 79646, Ukraine \\ e-mail: petkosob@gmail.com
}

Received: 15 January $2019 \quad$ Revised: 27 November 2019

Accepted: 9 March 2020

\begin{abstract}
In this paper it is shown that there is a plurality of irrational values of the roots of a quadratic equation with equal modulus coefficients $|p|=|q| \neq 1$ having properties of the numbers of Phidias $\varphi=0.61803 \ldots$ and $\Phi=1.61803 \ldots$ It is shown that it is also possible to construct a set of sequences possessing the basic properties of the Fibonacci and Lucas sequences.
\end{abstract}

Keywords: Golden ratio, Quadratic irrationality, Roots of quadratic equation.

2010 Mathematics Subject Classification: 11B37, 11 B39.

\section{Introduction}

It is known $[4,8,18]$ that the roots of a quadratic equation $(\mathrm{QE})$

$$
x^{2}+p x+q=0
$$

are irrational if the discriminant

$$
D=p^{2}-4 q
$$

is not equal to the exact square. For the values of coefficients $p=+1, q=-1$, equation (1) implies proportional equality

$$
x^{2}+x-1=x(x+1)-1=0 \Rightarrow \frac{x}{1}=\frac{1-x}{x},
$$

known as the proportion of the golden section of an integer $L=1$ into two unequal parts $x$ and $1-x$. When you split an integer $L$, the golden ratio is written as 


$$
\frac{x}{L}=\frac{L-x}{x}
$$

where, by introducing the coefficients of relative variable $L$

$$
\varphi=\frac{x}{L} \text { and } \Phi=\frac{L}{x}
$$

we get a system of QE

$$
\left\{\begin{array}{l}
\varphi^{2}+\varphi-1=0 \\
\Phi^{2}-\Phi-1=0
\end{array}\right.
$$

solutions are based on theorems Viete's, expressing the basic laws of the golden ratios in the form of equations:

$$
\left\{\begin{array}{c}
\left\{\begin{array} { l } 
{ \varphi _ { + } = + 0 . 6 1 8 \ldots , } \\
{ \varphi _ { - } = - 1 . 6 1 8 \ldots . }
\end{array} \Rightarrow \left\{\begin{array}{l}
\varphi_{+}+\varphi_{-}=-1, \\
\varphi_{+} \cdot \varphi_{-}=-1, \\
\varphi_{+}-\varphi_{-}=\sqrt{5},
\end{array} \Rightarrow \varphi_{+}{ }^{2}-\varphi_{-}{ }^{2}=-\sqrt{5} \Rightarrow \frac{1}{\varphi_{-}}+\frac{1}{\varphi_{+}}=1,\right.\right. \\
\left\{\begin{array} { l } 
{ \Phi _ { + } = + 1 . 6 1 8 \ldots , } \\
{ \Phi _ { - } = - 0 . 6 1 8 \ldots . . }
\end{array} \Rightarrow \left\{\begin{array}{l}
\Phi_{+}+\Phi_{-}=+1, \\
\Phi_{+} \cdot \Phi_{-}=-1, \\
\Phi_{+}-\Phi_{-}=\sqrt{5},
\end{array} \Rightarrow \Phi_{+}{ }^{2}-\Phi_{-}{ }^{2}=\sqrt{5} \Rightarrow \frac{1}{\Phi_{-}}+\frac{1}{\Phi_{+}}=-1 .\right.\right.
\end{array}\right.
$$

Quantitatively, the golden section is described by the positive values of the roots

$$
\varphi_{+}=\frac{1}{2}(-1+\sqrt{5})=0.618 \ldots=\varphi \text { and } \Phi_{+}=\frac{1}{2}(1+\sqrt{5})=1.618 \ldots=\Phi
$$

and for the positive values of the roots (4) by the Viete's theorem give:

$$
\left\{\begin{array}{l}
\Phi-\varphi=1, \\
\Phi \cdot \varphi=1,
\end{array} \Rightarrow \frac{1}{\varphi}-\frac{1}{\Phi}=1 .\right.
$$

Relations (9) express the main properties of the golden section.

This task was developed in many works, the results of which are systematized, for example, in monographs $[4,8,18]$ et al. The golden numbers $\Phi$ and $\varphi$ are currently used in modern research, quantum [21] and spin [1] physics, in new field of quasicrystals [14], fractal geometry [6], algebra of numbers [5, 16, 20]. The American mathematician G. Bergman [2] built a system of calculus, in which the number of Phidias plays the role of the basis. The publications $[3,12,13,16,17,19]$ indicate that the problem of proportional division remains relevant in the future. In this paper shows that the numbers $\Phi$ and $\varphi$ with "golden" properties (9) are not unique, and there is a set of irrational values of the roots of the generalized quadratic equation (1) with modulus coefficients equal to

$$
|p|=|q|=k \neq 1,
$$

with properties of Phidias numbers $\Phi$ and $\varphi$. 


\section{Theoretical model and discussion}

To substantiate this assertion, we formulate the division model (4) as

$$
\frac{x(k)}{L}=k \frac{L-x(k)}{x(k)}, k \neq 1,
$$

which has not been investigated recently in the literature, and introduce the coefficient of relative changes:

$$
\varphi(k)=\frac{x(k)}{L} \quad \text { and } \quad \Phi(k)=\frac{k}{\phi(k)} \cdot s
$$

Substituting Eq. (12) into Eq. (11), we obtain the square equation for $\varphi(k)$ and $\Phi(k)$ with solutions:

$$
\left\{\begin{array}{c}
\varphi(k)^{2}+k \cdot \varphi(k)-k=0 \Rightarrow\left\{\begin{array}{c}
\varphi_{ \pm}(k)=\frac{k}{2}(-1 \pm \sqrt{1+4 / k}), \\
\varphi_{+}(k)+\varphi_{-}(k)=-k, \\
\varphi_{+}(k) \cdot \varphi_{-}(k)=-k,
\end{array} \Rightarrow \frac{1}{\varphi_{+}(k)}+\frac{1}{\varphi_{-}(k)}=1,\right. \\
\Phi_{ \pm}(k)=\frac{k}{2}(1 \pm \sqrt{1+4 / k}), \\
\Phi(k)^{2}-k \Phi(k)-k=0 \Rightarrow\left\{\begin{array}{c}
\Phi_{+}(k)+\Phi_{-}(k)=+k, \\
\Phi_{+}(k) \cdot \Phi_{-}(k)=-k,
\end{array} \Rightarrow \frac{1}{\Phi_{+}(k)}+\frac{1}{\Phi_{-}(k)}=-1,\right.
\end{array}\right.
$$

similar to the gold ones for numbers $\varphi$ and $\Phi(7)$, as confirmed in Table 1 by calculations the roots (13) for arbitrarily taken integers, fractional and irrational values of the coefficient $k$ [10].

\begin{tabular}{|l|l|l|l|l|}
\hline \multicolumn{1}{|c|}{$k$} & $\Phi_{+}(k)$ & $\varphi_{+}(k)$ & $\Phi_{-}(k)$ & $\varphi_{-}(k)$ \\
\hline 1 & $1.618 \ldots$ & $0.618 \ldots$ & $-0.618 \ldots$ & $-1.618 \ldots$ \\
\hline 2 & $2.732 \ldots$ & $0.732 \ldots$ & $-0.732 \ldots$ & $-2.732 \ldots$ \\
\hline$\pi=3.14 \ldots$ & 3.937 & 0.797 & -0.937 & -3.937 \\
\hline 11 & $11.923 \ldots$ & $0.923 \ldots$ & $-0.923 \ldots$ & $-11.923 \ldots$ \\
\hline
\end{tabular}

Table 1. Numerical values of roots calculated by formulas (13)

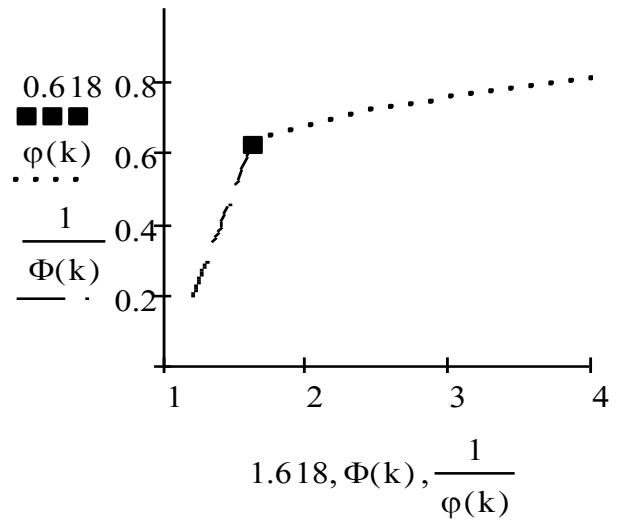

Figure 1. Phase diagram roots $\varphi(k), \Phi(k)$ 
The graphs of dependency (13) for positive solutions for positive solutions of $\varphi(k)=\varphi_{+}(k)$ and $\Phi(k)=\Phi_{+}(k)$ are shown in Figure 1. The fact that the graphs of both solutions converge to a common point indicates the correctness of the proposed model of generalization of the proportional division of the whole into two unequal parts in the phase direction (10), which on the Cartesian plane $p 0 q$ is parallel to the direction of the root line $p+q+1=0$ [9].

\section{The set of sequences with the properties of the Fibonacci and Lucas numbers}

Members of the Fibonacci sequence [4, 8, 18]

$$
F_{n+1}=F_{n}+F_{n-1}, F(0)=1, F(1)=1, n=1,2,3,4,5, \ldots
$$

and members of the Lucas sequence

$$
L_{n}=L_{n-1}+L_{n}, L(0)=1, L(1)=3, n=1,2,3,4,5, \ldots
$$

are related to the numbers $\Phi, \varphi$ by Binet formulas [4]:

$$
F_{n}=\frac{1}{\sqrt{5}}\left(\Phi^{n}-(-1)^{n} \Phi^{-n}\right)(a) \text { and } L_{n}=\Phi^{n}+(-1)^{n} \Phi^{-n}(b)
$$

taking into account the Cassini formula

$$
F_{n+1} \cdot F_{n-1}-F_{n}^{2}=(-1)^{n} \quad \text { for } \quad n \geq 1 .
$$

We formulate by the analogy the Binet relations, which allow us to construct with the help of numbers $\Phi(k)$ and $\varphi(k)$ sequences:

$$
F_{k, n}=\frac{\Phi(k)^{n}-(-1)^{n} \varphi(k)^{n}}{\sqrt{k^{2}+4 k}}(a) \text { and } L_{k, n}=\Phi(k)^{n}+(-1)^{n} \Phi(k)^{n}(b)
$$

for which recurrence relations are valid

$$
F_{k, n+2}=\left(F_{k, n+1}+F_{k, n}\right) k \quad \text { and } \quad L_{k, n+2}=\left(L_{k, n+1}+L_{k, n}\right) k, \quad F_{k, 1}=1, F_{k, 2}=k,
$$

Cassini formula

$$
\left\lfloor F_{k, n+1} \cdot F_{k, n-1}-F_{k, n}^{2}\right\rfloor \cdot k=(-k)^{n} \quad \text { for } \quad n \geq 1 .
$$

The formulas (18)-(20) written down are proved by induction. Indeed, when $k=1$ we have:

$$
\left\{\begin{array}{l}
F_{n}=\frac{\Phi^{n}-(-1)^{n} \varphi^{n}}{\sqrt{5}} \\
L_{n}=\Phi^{n}+(-1)^{n} \Phi^{n}
\end{array} \Rightarrow \Phi^{n}-F_{n} \sqrt{5}=L_{n}-\Phi^{n} \Rightarrow \Phi^{n}=\frac{1}{2}\left(L_{n}+F_{n} \sqrt{5}\right) .\right.
$$

Consequently, the formulas (18)-(20) are valid. The basis of the induction is proved. 
By induction, any degree $n$ of the golden proportion $\Phi(k)$ through Lucas numbers $L_{k, n}$ and the Fibonacci $F_{k, n}$ numbers can be expressed as:

$$
\Phi(k)^{n}=\frac{1}{2}\left(L_{k, n}+F_{k, n} \sqrt{k^{2}+4 k}\right) .
$$

The correctness of (18)-(20) is confirmed by the fact that formula (18) is followed by an analytical expression for calculating the terms of the Fibonacci sequence $u_{n}$ [11] for the GP model

$$
\frac{L}{x}=\alpha^{2} \frac{x}{L-x} .
$$

The results of calculations of the Fibonacci numbers and the Lucas numbers are presented in Table 3. As follows from Tables 2 and 3, the values of the ratios

$$
\frac{F_{k, n+1}}{F_{k, n}} \text { and } \frac{L_{k, n+1}}{L_{k, n}}
$$

fluctuate relative to the values of the gold numbers $\Phi(k)$, tending to them as equilibrium for the given GP numbers $\Phi(1)=\Phi=1.618 . ., \Phi(2)=2.732 . ., \Phi(\pi)=3.937 \ldots$, etc. [7] .

\begin{tabular}{|l|l|l|l|l|l|}
\hline$F(1, n)$ & $\frac{F(1, n+1)}{F(1, n)}$ & \multicolumn{1}{|c|}{$F(2, n)$} & $\frac{F(2, n+1)}{F(2, n)}$ & $F(\pi, n)$ & $\frac{F(\pi, n+1)}{F(\pi, n)}$ \\
\hline 1 & & 1 & & 1 & \\
\hline 1 & 1 & 2 & 2 & $\pi$ & 3.14 \\
\hline 2 & 2 & $6=(2+1) \times 2$ & 3 & $13=(\pi+1) \times \pi$ & 4.14 \\
\hline 3 & 1.5 & $16=(6+2) \times 2$ & 2.667 & $50.7=(13+\pi) \times \pi$ & 3.898 \\
\hline 5 & 1.667 & $44=(16+6) \times 2$ & 2.75 & $199.95=(50.7+13) \times \pi$ & 3.945 \\
\hline 8 & 1.6 & $120=(44+16) \times 2$ & 2.733 & $786.99=(200+50.7) \times \pi$ & 3.936 \\
\hline 13 & 1.625 & $328=(120+44) \times 2$ & 2.732 & $3099=(787+200) \times \pi$ & 3.938 \\
\hline 21 & 1.619 & $8952=(328+120) \times 2$ & 2.732 & $12200=(3099+787) \times \pi$ & 3.937 \\
\hline
\end{tabular}

Table 2. The numerical values of the members of the Fibonacci sequence and its ratio

\begin{tabular}{|l|l|l|l|l|l|l|}
\hline$n$ & \multicolumn{1}{|c|}{$L(2, n)$} & $\frac{L(2, n)}{L(2, n-1)}$ & $\frac{L(2, n-1)}{L(2, n)} \cdot k$ & \multicolumn{1}{|c|}{$L(\pi, n)$} & $\frac{L(\pi, n)}{L(\pi, n-1)} \rightarrow 3.94$ & $\frac{L(\pi, n-1)}{L(\pi, n)} \cdot \pi \rightarrow 0.798$ \\
\hline 0 & $L(2,0)=2$ & & & $L(\pi, 0)=2$ & & \\
\hline 1 & $L(2,1)=2$ & & & $L(\pi, 1)=\pi$ & & \\
\hline 2 & $8=(2+2) \times 2$ & 4 & 0.5 & $16.153=(\pi+2) \times \pi$ & 5.14 & 0.611 \\
\hline 3 & $20=(8+2) \times 2$ & 2.5 & 0.8 & $60.615=(16+\pi) \times \pi$ & 3.753 & 0.837 \\
\hline 4 & $56=(20+8) \times 2$ & 2.8 & 0.714 & $241=(60.5+16) \times \pi$ & 3.98 & 0.79 \\
\hline 5 & $152=(56+20) \times 2$ & 2.714 & 0.737 & $948.1=(241+61) \times \pi$ & 3.93 & 0.797 \\
\hline 6 & $416=(152+56) \times 2$ & 2.737 & 2.731 & $3736=(946+241) \times \pi$ & 3.94 & 0.798 \\
\hline
\end{tabular}

Table 3. Numeric values of members of Luke sequence numbers and its ratio 


\section{Conclusion}

The irrational roots of the quadratic equation $x^{2}+p x+q=0$ with equal modulus $|p|=|q|=k \neq 1$ having properties of the numbers of Phidias $\varphi=0.61803 \ldots$ and $\Phi=1.61803 \ldots$ The general model of golden section have the form

$$
\frac{x(k)}{L}=k \frac{L-x(k)}{x(k)}
$$

for arbitrary values of the coefficient $k$. On the basic of quadratic irrationality can by constructive a set of sequences with the properties of the terms of the sequences Fibonacci and Lucas.

\section{References}

[1] Affleck, I. (2010). Solid-state physics: golden ratio seen in a magnet. Nature, 464 (7287), 362-363.

[2] Bergman, G. (1957). A number system with an irrational base. Math. Magazine, 31, 98-110.

[3] Bradley, S. (2000). A geometric connection between generalized Fibonacci sequences nearly golden section. The Fibonacci Quart., 38 (2), 174-179.

[4] Dunlap, R. (1997). The Golden Ratio and Fibonacci Numbers. World Scientific Publishing Co. Pte. Ltd.

[5] Finch, S. Mathematical Constants. Cambridge University Press, 2003.

[6] Franz, M. (2009). A Fractal Made Of Golden Sets, Mathematics Magazine, 82, 243-254.

[7] Horadam, A., \& Shannon, A. G. (1988). Fibonacci and Lucas Curves, Fibonacci Quart., $26(1), 3-13$.

[8] Koshy, T. (2001). Fibonacci and Lucas Numbers with Application, A Wiley-Interscience Publication: New York.

[9] Kosobutskyy, P., \& Karkulovska, M. (2019). Mathematical methods for CAD: The method of proportional division. Bulletin of the Lviv Polytechnic National University. Collection of scientific works. Scientific publication. Series: Computer Design Systems. Theory and practice. 908, 75-83.

[10] Kosobutskyy, P. S. (2018). On the Possibility of Constructing a Set of Numbers with Golden Section Properties. International Conference Algebra and Analysis with Application. July 1-4 2018, Ohrid, Republic of Macedoni.

[11] Krcadinac, V. (2006). A new generalization of the golden ratio. Web Resource: https://www.fq.math.ca/Papers1/44-4/quartkrcadinac04_2006.pdf.

[12] Leyendekkers, J. V., \& Shannon, A. G. (2016). Some Golden Ratio generalized Fibonacci and Lucas sequences. Notes on Number Theory and Discrete Mathematics, 22 (1), 33-41. 
[13] Leyendekkers, J. V., \& Shannon, A. G. (2018). Generalized golden ratios and associated Pell sequences. Notes on Number Theory and Discrete Mathematics, 24 (3), 103-110.

[14] Shechtman, D., Blech, I., Gratias, D., \& Cahn, J. W. (1984). Metallic phase with longrange orientational order and no translational symmetry. Phys. Rev. Lett., 53, 1951-1953.

[15] Shneider, R. (2016). Fibonacci numbers and the golden ratio. Web Resource: arXiv:1611.07384v1 [math.HO] 22 Nov 2016.

[16] Shneider, R. (2016). Fibonacci numbers and the golden ratio. Website.com, Available online at: arXiv:1611.07384v1 [math.HO] 22 Nov 2016.

[17] Szakacs, T. (2017). $k$-order linear recursive sequences and the golden ratio. The Fibonacci Quart., 55 (5), 186-191.

[18] Vajda, S. (1989). Fibonacci \& Lucas Numbers, and the Golden Section. Theory and Applications. Ellis Horwood limited.

[19] Vernon, R. P. (2018). Relationships between Fibonacci-type sequences and Golden-type ratios. Notes on Number Theory and Discrete Mathematics, 24 (2), 85-89.

[20] Yiu, P. (2003). Recreational Mathematics. Department of Mathematics Florida Atlantic University. Web Resource: http://math.fau.edu/yiu/RecreationalMathematics 2003.pdf.

[21] Yu, D., Xue D., \& Ratajczak, H. (2006). Golden ratio and bond-valence parameters of hydrogen bonds of hydrated borates. Journal of Molecular Structure, 783 (1-3), 210-214. 